\title{
Inhibitory action of C22-fatty acids on DNA polymerases and DNA topoisomerases
}

\author{
YUKO YONEZAWA ${ }^{1,2}$, TAKAHIKO HADA ${ }^{3}$, KEISUKE URYU ${ }^{3}$, \\ HIROSHI IIJIMA $^{1}$, HIROMI YOSHIDA ${ }^{1,2}$ and YOSHIYUKI MIZUSHINA ${ }^{1,2}$ \\ ${ }^{1}$ Laboratory of Food and Nutritional Sciences, Department of Nutritional Science; ${ }^{2}$ Cooperative Research Center \\ of Life Sciences, Kobe-Gakuin University, Nishi-ku, Kobe, Hyogo 651-2180; ${ }^{3}$ Department of Research \\ and Development, Bizen Chemical Co., Ltd., Tokutomi, Akaiwa, Okayama 709-0716, Japan
}

Received April 3, 2006; Accepted June 2, 2006

\begin{abstract}
We reported previously that unsaturated linear-chain fatty acids of the cis-configuration with a C18-hydrocarbon chain such as linoleic acid (cis-9, 12-octadecadienoic acid, C18:2) could potently inhibit the activity of mammalian DNA polymerases (Biochim Biophys Acta 1308: 256-262, 1996). In this study, we investigated the inhibitory effects of cis-type C22-fatty acids including cis-7,10,13,16,19-docosapentaenoic acid (DPA, C22:5) and cis-4,7,10,13,16,19-docosahexaenoic acid (DHA, C22:6) on mammalian DNA polymerases and human DNA topoisomerases. Cis-13,16-docosadienoic acid (C22:2) was the strongest inhibitor of both DNA polymerases and topoisomerases of all C22-fatty acids tested. The inhibitory tendency by the fatty acids on DNA polymerases was the same as that of DNA topoisomerases, and the second strongest inhibitor was cis-13,16,19-docosatrienoic acid (C22:3). The energy-minimized three-dimensional structures of the fatty acids were calculated and it was found that a length of 19-21 and width of more than $7 \AA$ in C22-fatty acid structure were important for enzyme inhibition. The three-dimensional structure of the active site of both DNA polymerases and topoisomerases must have a pocket to join $\mathrm{C} 22: 2$, and this pocket was $19.41 \AA$ long and $9.58 \AA$ wide.
\end{abstract}

Correspondence to: Dr Yoshiyuki Mizushina, Laboratory of Food and Nutritional Sciences, Department of Nutritional Science, KobeGakuin University, Nishi-ku, Kobe, Hyogo 651-2180, Japan

E-mail:mizushin@nutr.kobegakuin.ac.jp

Abbreviations: pol, DNA-directed DNA polymerase (E.C.2.7.7.7); Topo, DNA topoisomerase; PUFA, polyunsaturated fatty acids; DMSO, dimethyl sulfoxide; $\mathrm{IC}_{50}, 50 \%$ inhibitory concentration; poly(dA), polydeoxyriboadenylic acid; oligo(dT) ${ }_{12-18}$, oligo(12-18) deoxyribothymidylic acid; dTTP, 2'-deoxythymidine 5'-triphosphate; dsDNA, double-stranded DNA; BSA, bovine serum albumin

Key words: C22-fatty acid, cis-13,16-docosadienoic acid (C22:2), DNA polymerase, DNA topoisomerase, enzyme inhibitor

\section{Introduction}

We have studied the in vitro and in vivo ability of mammalian DNA metabolic enzymes, especially DNA polymerases (pols), to use these selective inhibitors, and established an assay to detect pol inhibitors $(1,2)$. We screened the products of microbial fermentation for those which inhibit pol activity. We found an inhibitor from a bisidiomycete, a well-known fatty acid, linoleic acid (cis-9,12-octadecadienoic acid, C18:2) (2). Subsequently, we investigated the effects of commercially available fatty acids on the activity of pols. We found that several fatty acids, particularly long chain fatty acids with a cis-configuration, interact with pols and suppress their activity. In this manuscript, we discuss the effects of C22-fatty acids including well-known bioactive compounds such as cis-7,10, 13,16,19-docosapentaenoic acid (DPA, C22:5) and cis-4,7, 10,13,16,19-docosahexaenoic acid (DHA, C22:6), which are polyunsaturated fatty acids (PUFA), on the activity of DNA metabolic enzymes such as pols and DNA topoisomerases (topos).

Eukaryotic cells reportedly contain three replicative pols, $\alpha, \delta$, and $\varepsilon$, mitochondrial pol $\gamma$ and at least twelve repair types of pols, $\beta, \delta, \varepsilon, \zeta, \eta, \theta, \iota, \kappa, \lambda, \mu, \sigma$, and $\phi(3,4)$. Pol catalyzes the addition of deoxyribonucleotides to the 3 '-hydroxyl terminus of primed double-stranded DNA molecules (3).

Topos are key enzymes that control the topological state of DNA, and topo catalyzes the concerted breaking and rejoining of DNA strands and is involved in producing the necessary topological and conformational changes in DNA $(3,5)$. There are two classes of topos: type I enzymes, which act by transiently nicking one of the two DNA strands, and type II enzymes, which nick both DNA strands, are dependent on ATP, and are involved in many vital cellular processes that influence DNA replication, transcription, recombination, integration and chromosomal segregation (3). In recent years, these enzymes have received special interest because topo inhibitors have emerged as anti-cancer (6) and anti-parasitic agents $(7,8)$. Topos can be inhibited by two distinct mechanisms, and the inhibitors are divided into two classes accordingly: classes I and II. Class I inhibitors stabilize the enzyme-DNA covalent complex and block the subsequent rejoining of the DNA break. Class II inhibitors, also referred 
to as catalytic inhibitors, prevent the enzyme and DNA from binding by interacting with either the topo $(9,10)$ or DNA (11).

Therefore, pol and topo are critical to many cellular processes such as DNA replication, repair and recombination, and may act in harmony. We suggest the three-dimensional site where the enzyme binds to C22-fatty acids using computer modeling and three-dimensional structural analysis. The molecular design method may be useful to create agents for cancer chemotherapy.

\section{Materials and methods}

Materials. Saturated C22-fatty acid, $n$-docosanoic acid (behenoic acid, C22:0), the cis-configuration of unsaturated C22-fatty acids such as cis-13-docosenoic acid (erucic acid, C22:1), cis-13,16-docosadienoic acid (C22:2), cis-13,16,19docosatrienoic acid (C22:3), cis-7,10,13,16-docosatetraenoic acid (adrenic acid, C22:4), cis-7,10,13,16,19-docosapentaenoic acid (DPA, C22:5) and cis-4,7,10,13,16,19-docosahexaenoic acid (DHA, C22:6) were purchased from Nu-Chek-Prep Inc. (Minnesota, USA). Nucleotides such as $\left[{ }^{3} \mathrm{H}\right]-2^{2}-$ deoxythymidine 5 '-triphosphate (dTTP) $(43 \mathrm{Ci} / \mathrm{mmol})$, and chemically synthesized template-primers such as poly(dA) and oligo(dT) $)_{12-18}$ were purchased from Amersham Biosciences (Buckinghamshire, UK). All other reagents were of analytical grade and were purchased from Nacalai Tesque Ltd. (Kyoto, Japan).

Enzymes. Pol $\alpha$ was purified from calf thymus by immunoaffinity column chromatography as described by Tamai et al (12). Recombinant rat pol $\beta$ was purified from E. coli JMp 35 as described by Date et al (13). Human pol $\delta$ and $\varepsilon$ were purified from the nuclear fraction of human peripheral blood cancer cells (Molt-4) using the second subunit of pol $\delta$ and $\varepsilon$ conjugated affinity column chromatography, respectively (14). Recombinant human His-pol $\lambda$ was overexpressed and purified according to a method described by Shimazaki et al (15). Human recombinant topo I and topo II ( 2 units/ $\mu 1$ each) were purchased from TopoGen, Inc. (Columbus, OH, USA).

DNA polymerase assays. The reaction mixtures for pols $\alpha$ and $\beta$ were described previously $(1,2)$, and those for pols $\delta$ and $\varepsilon$ were described previously (16). The reaction mixture for pol $\lambda$ was the same as that for pol $B$. For pols, poly $(\mathrm{dA}) /$ oligo $(\mathrm{dT})_{12-18}$ and dTTP (2'-deoxythymidine 5'-triphosphate) were used as the template-primer DNA and nucleotide substrate, respectively. C22-fatty acids were dissolved in dimethyl sulfoxide (DMSO) at various concentrations and sonicated for $30 \mathrm{sec}$. Aliquots of $4 \mu \mathrm{l}$ of sonicated samples were mixed with $16 \mu 1$ of each enzyme (final, 0.05 units) in $50 \mathrm{mM}$ Tris- $\mathrm{HCl}(\mathrm{pH} 7.5)$ containing $1 \mathrm{mM}$ dithiothreitol, $50 \%$ glycerol and $0.1 \mathrm{mM}$ EDTA, and kept at $0^{\circ} \mathrm{C}$ for $10 \mathrm{~min}$. These inhibitor-enzyme mixtures $(8 \mu \mathrm{l})$ were added to $16 \mu \mathrm{l}$ of each enzyme standard reaction mixture, and incubation was carried out at $37^{\circ} \mathrm{C}$ for $60 \mathrm{~min}$. Activity without the inhibitor was considered $100 \%$, and the remaining activity at each concentration of the inhibitor was determined relative to this value. One unit of pol activity was defined as the amount of enzyme that catalyzed the incorporation of $1 \mathrm{nmol}$ of
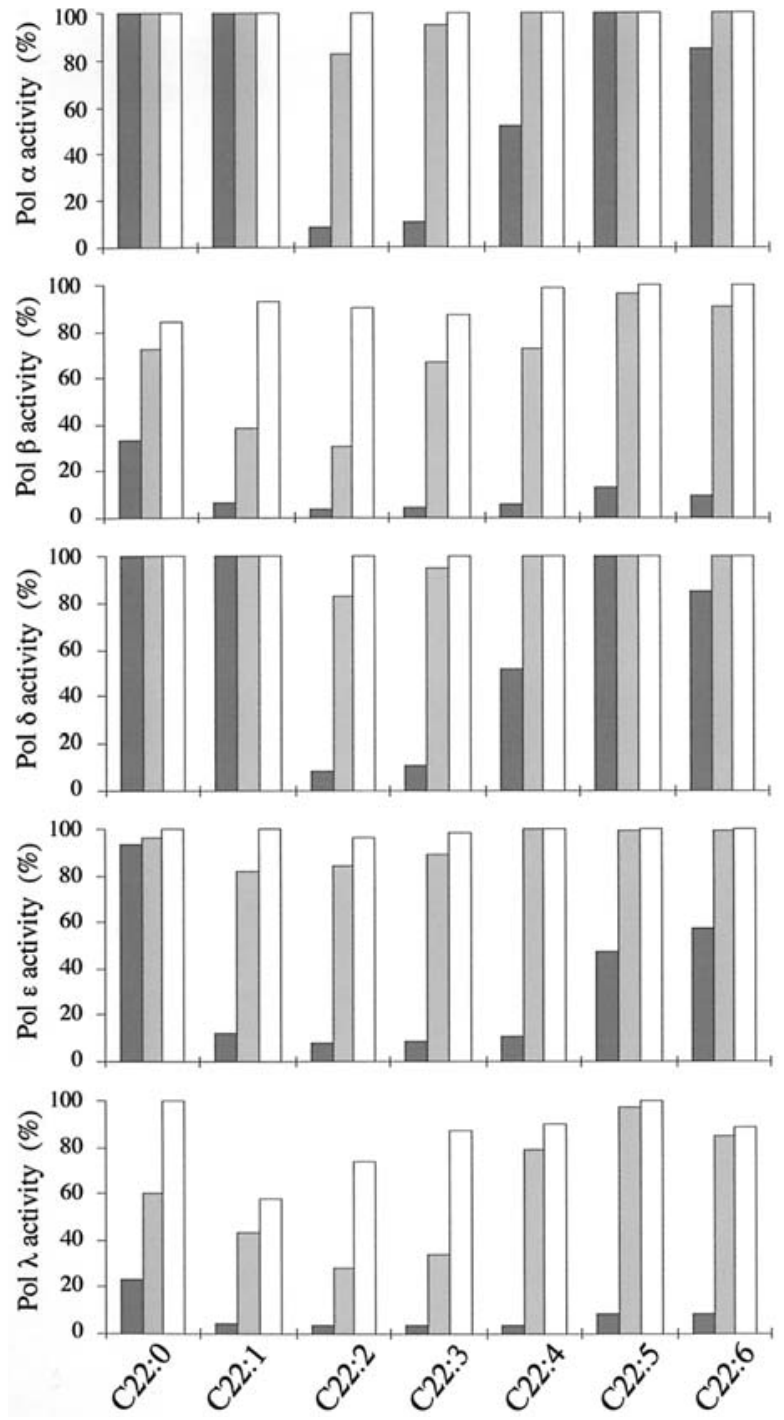

Figure 1. Inhibitory effect of linear-chain C22-fatty acids on mammalian DNA polymerases. The investigated pols are calf pol $\alpha$, rat pol $\beta$, human pol $\delta$, human pol $\varepsilon$, human pol $\lambda$. The dark-gray bars, light-gray bars and whitebars are 100,10 and $1 \mu \mathrm{M}$ of C22-fatty acids, respectively. Each pol is 0.05 units. Enzyme activity in the absence of fatty acids was taken as $100 \%$.

deoxyribonucleotide triphosphates (i.e., dTTP) into synthetic template-primers [i.e. poly $(\mathrm{dA}) / \mathrm{oligo}(\mathrm{dT})_{12-18}, \mathrm{~A} / \mathrm{T}=2 / 1$ ] in $60 \mathrm{~min}$ at $37^{\circ} \mathrm{C}$ under normal reaction conditions for each enzyme (2).

DNA topoisomerase assays. Relaxation activity of topos was determined by detecting the conversion of supercoiled plasmid DNA to its relaxed form. The topo II reaction was performed in 20- $\mu 1$ reaction mixtures containing $50 \mathrm{mM}$ Tris- $\mathrm{HCl}$ buffer (pH 8.0), $120 \mathrm{mM} \mathrm{KCl}, 10 \mathrm{mM} \mathrm{MgCl} 2,0.5 \mathrm{mM}$ ATP, $0.5 \mathrm{mM}$ dithiothreitol, pBR322 plasmid DNA (200 ng), $2 \mu 1$ of inhibitor solution (10\% DMSO) and 1 unit of topo II. The reaction mixtures were incubated at $37^{\circ} \mathrm{C}$ for $30 \mathrm{~min}$ and terminated by adding $2 \mu \mathrm{l}$ of loading buffer consisting of 5\% sarkosyl, $0.0025 \%$ bromophenol blue and $25 \%$ glycerol. The mixtures were subjected to $1 \%$ agarose gel electrophoresis in TAE (Trisacetate-EDTA) running buffer. The agarose gels were stained with ethidium bromide, and DNA was visualized on a UV transilluminator. Zero-D scan (version 1.0, M\&S Instruments 


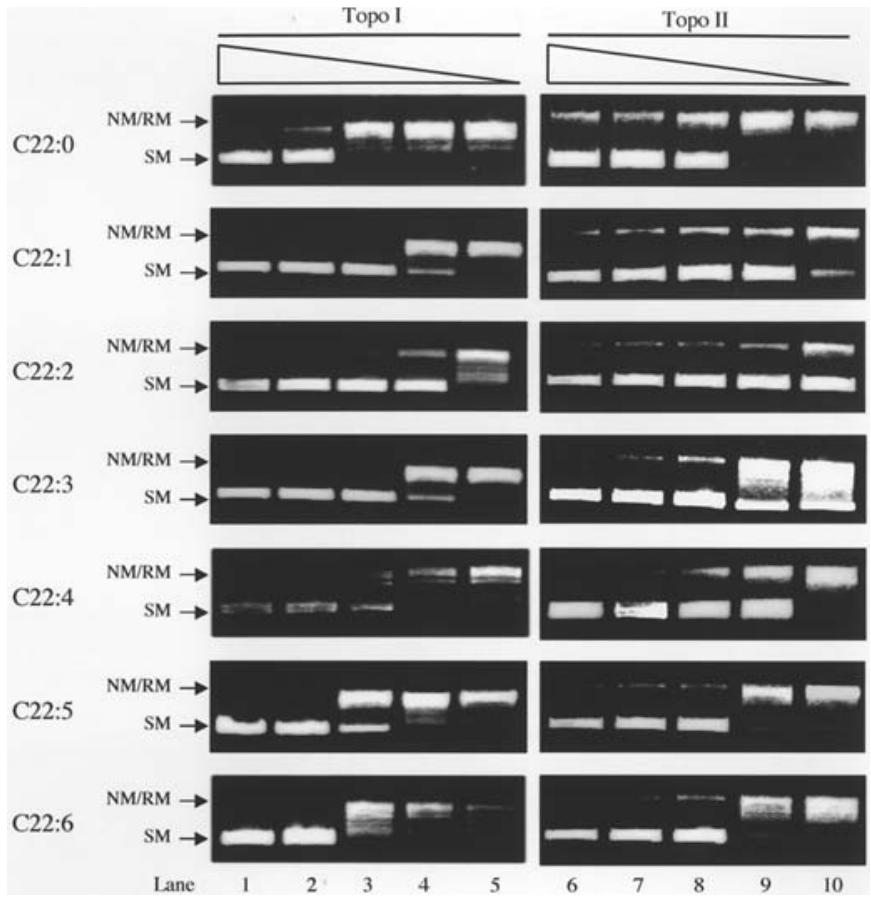

Figure 2. Inhibition of relaxation activity of human DNA topoisomerases by C22-fatty acids. Lanes 1-5 and lanes 6-10 had topo I (2 units) and topo II ( 2 units) added to the reaction mixture, respectively. Lanes 1 and 6,2 and 7, 3 and 8,4 and 9 , and 5 and 10 had C-22 fatty acids at concentrations of 25, $5,1,0.2$, and $0.04 \mu \mathrm{M}$, respectively; $200 \mathrm{ng}$ of plasmid DNA was added to each of the lanes. Photographs of ethidium bromide-stained gels are shown.

Trading Inc., Tokyo, Japan) was used for densitometric quantitation of the plasmid DNA products. The relaxation activity of topo I was analyzed in the same manner as described above except that reaction mixtures contained $10 \mathrm{mM}$ Tris- $\mathrm{HCl}$ (pH 7.9), pUC19 plasmid DNA (200 ng), 1 mM EDTA, $150 \mathrm{mM} \mathrm{NaCl}, 0.1 \%$ bovine serum albumin (BSA), $0.1 \mathrm{mM}$ spermidine, $5 \%$ glycerol and 1 unit of topo I. One unit was defined as the amount of enzyme capable of relaxing $0.25 \mu \mathrm{g}$ of DNA in $15 \mathrm{~min}$ at $37^{\circ} \mathrm{C}$.

Computational analysis of C22-fatty acids. A compound model was constructed and simple-minimized. Compound models were simulated with force field parameters based on the consistent valence force field (CVFF). Group-based cutoffs, $0.95 \mathrm{~nm}$ for van der Waals and $0.95 \mathrm{~nm}$ for Coulomb interactions, were introduced. The temperature was set at 298 K. Calculations based on simulation images were carried out using Insight II (Accelrys Inc., San Diego, CA, USA).

\section{Results}

Inhibitory effects of C22-fatty acids on the activity of mammalian DNA polymerases. First, we tested whether linear-chain C22-fatty acids have the ability to inhibit representative nuclear pols such as calf pol $\alpha$, rat pol $\beta$, human pol $\delta$, human pol $\varepsilon$ and human pol $\lambda$. Pols $\alpha, \delta$ and $\varepsilon$ are replicative pols, and pols $\beta$ and $\lambda$ are repair-related pols $(3,4)$. One, 10 and $100 \mu \mathrm{M}$ of fatty acids with a 22-hydrocarbon chain were tested (Fig. 1). Inhibition by these C22-fatty acids was dose-dependent, and the inhibitory effect had the same tendency among the fatty acids. In the $\mathrm{IC}_{50}$ values and the relative effect on pols $\alpha-\lambda$ by $100 \mu \mathrm{M}$ of compounds, cis13,16-docosadienoic acid (C22:2) was the strongest inhibitor of all C22-fatty acids tested, and cis-13,16,19-docosatrienoic acid (C22:3) was the second strongest. Since the inhibitory effect of saturated C22-fatty acid, $n$-docosanoic acid (C22:0), was weaker than that of C22-PUFAs, the cis-configuration of double bonds must be important for pol inhibition. In the order of pol effect by $\mathrm{C} 22$-fatty acids, the inhibition of pols $\beta$ and $\lambda$ was stronger than that of pols $\alpha, \delta$ and $\varepsilon$, because the $\mathrm{IC}_{50}$ values of cis-13,16-docosadienoic acid (C22:2) for pols $\alpha, \beta$, $\delta$, $\varepsilon$ and $\lambda$ were $47,6.0,46,47$ and $3.0 \mu \mathrm{M}$, respectively. These results suggested that $\mathrm{C} 22$-fatty acids, especially cis-13,16docosadienoic acid (C22:2), were more potent inhibitors for repair-related pols than replicative pols.

Inhibitory effects of C22-fatty acids on the activity of human DNA topoisomerases. Next, we also investigated the inhibitory activity of human topos I and II instead of mammalian pols. Pols and topos are DNA metabolic enzymes, and both inhibitors could be anti-cancer agents. As shown in Fig. 2, C22-fatty acids dose-dependently inhibited the activity of topo I and topo II. The inhibitory effect of topo I had the same tendency as that of topo II, and in the order of their effect, C22-fatty acids ranked as follows: C22:2 > C22:3 > C22:1 > $\mathrm{C} 22: 4>\mathrm{C} 22: 5>\mathrm{C} 22: 6>\mathrm{C} 22: 0$. This ranking was almost the same as the inhibitory effect for mammalian pols (Fig. 1), suggesting that pols and topos might have the same inhibitory mechanism, although their modes of action, amino acid sequences and 3D structures are markedly different $(3,5)$. The $\mathrm{IC}_{50}$ values of cis-13,16-docosadienoic acid (C22:2) for topos I and II were 0.1 and $0.03 \mu \mathrm{M}$, respectively, suggesting that the effect of topo II was stronger than that of topo I. The inhibition of topo II by C22-fatty acids was approximately 200 -fold, 1,500-fold stronger than that of replicative pols such as pols $\alpha, \delta$ and $\varepsilon$, and repair-related pols such as pols $B$ and $\lambda$, respectively.

Double reciprocal plots of the results indicated that the inhibition of both calf pol $\alpha$, rat pol $\beta$ and human topos I and II by cis-13,16-docosadienoic acid (C22:2) was through competition with the substrate DNA (data not shown), suggesting that C22-fatty acids might compete with the DNA to bind to the catalytic site of the pols and topos.

C22-fatty acids did not inhibit the activity of other DNAmetabolic enzymes such as the primase of calf pol $\alpha, \mathrm{HIV}-1$ reverse transcriptase, T7 RNA polymerase, T4 polynucleotide kinase or bovine deoxyribonuclease I (data not shown). The results suggested that these compounds could selectively inhibit the activity of mammalian pols and human topos.

When the carboxyl group of C22-fatty acid was chemically modified resulting in a methyl-ester, ethyl-ester, alcohol, or completely removed, C22-hydrocarbon (i.e., docosane) did not inhibit the activity of pols and topos (data not shown), showing the importance of the free carboxyl group for inhibition. The inhibitory effect of the trans-configuration of C22-fatty acid such as trans-13-docosenoic acid (brassidic acid, trans-C22:1) was significantly weaker than that of cistype. These results suggested that cis-type unsaturated C22fatty acids such as cis-13,16-docosadienoic acid (C22:2) with free carboxylate were potent inhibitors of these enzymes. 


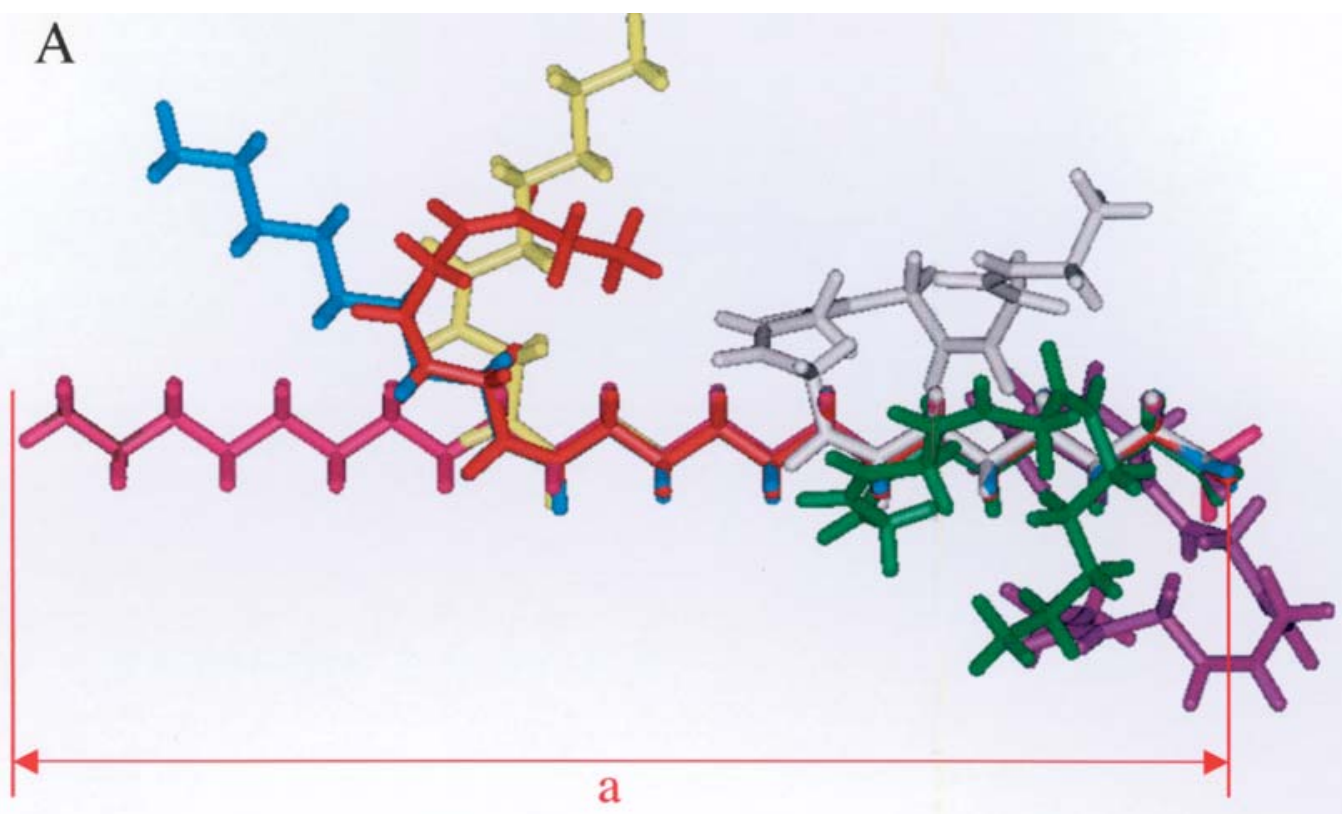

B

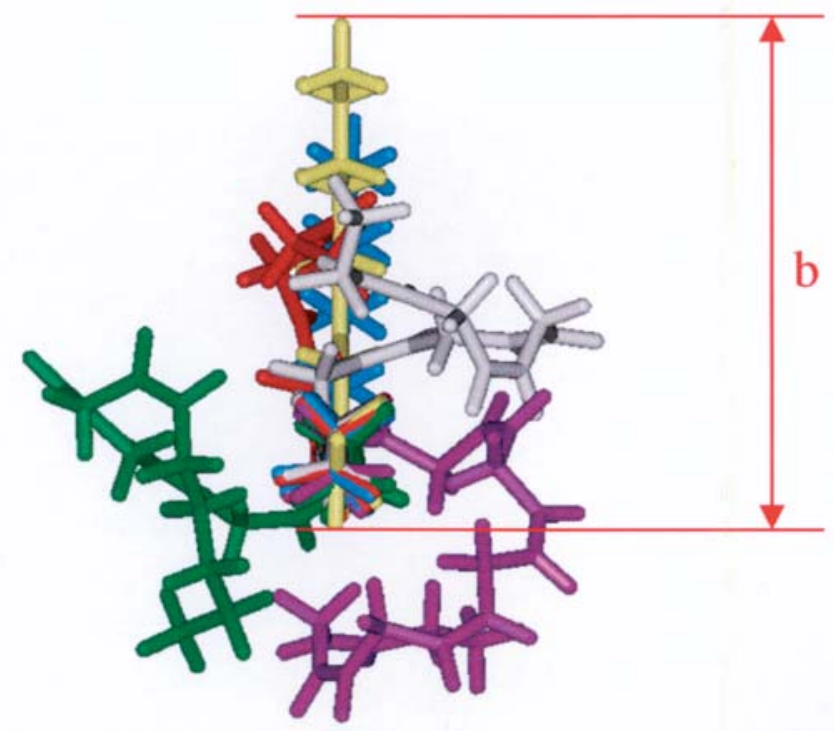

Figure 3. Computer graphics of the overlay of linear-chain C22-fatty acids. (A) The colors of C22-fatty acids are as below: $n$-docosanoic acid (C22:0, violet), the cis-configuration of unsaturated C22-fatty acids such as cis-13-docosenoic acid (C22:1, light-blue), cis-13,16-docosadienoic acid (C22:2, yellow), cis13,16,19-docosatrienoic acid (C22:3, red), cis-7,10,13,16-docosatetraenoic acid (C22:4, green), cis-7,10,13,16,19-docosapentaenoic acid (C22:5, light-gray) and cis-4,7,10,13,16,19-docosahexaenoic acid (C22:6, blue). Both carbon atoms in the carboxyl group and the hydrocarbon chain of their fatty acids were joined. (B) The right side of A is shown. The length (a) and width (b) of the fatty acid molecular structure are shown. Prepared using Insight II (Accelrys, San Diego, CA, USA).

Inhibition mechanism of C22-fatty acids on DNA polymerases and topoisomerases. To determine whether the inhibitor resulted in binding to DNA or the enzyme, the interaction of C22-fatty acids with double-stranded DNA (dsDNA) was investigated based on the thermal transition of dsDNA with or without C22-fatty acids. The Tm of dsDNA with an excess amount of $\mathrm{C} 22$-fatty acids $(100 \mu \mathrm{M})$ was measured using a spectrophotometer equipped with a thermoelectric cell holder. In the concentration range used, no thermal transition of Tm was observed, whereas ethidium bromide as a positive control, a typical intercalating compound, produced a clear thermal transition. These results indicated that $\mathrm{C} 22$-fatty acids did not intercalate to dsDNA as a substrate, and the compounds might directly bind to the enzyme and inhibit its activity.

To determine the effects of a non-ionic detergent on the binding of C22-fatty acids to pols and topos, Nonidet P-40 (NP-40) was added to the reaction mixture at a concentration of $0.1 \%$. In the absence of C22-fatty acids, the activity of pols and topos was not affected by the addition of NP-40, and we designated the activity in these cases as $100 \%$. The inhibitory effect of C22-fatty acids at $100 \mu \mathrm{M}$ was largely reversed by the addition of $0.1 \%$ NP- 40 to the reaction mixture. 
Table I. The molecular length and width of the threedimensional structure of C22-fatty acids.

\begin{tabular}{lcc}
\hline Fatty acid & Length $(\AA)$ & Width $(\AA)$ \\
\hline C22:0 & 28.86 & - \\
C22:1 & 27.15 & 6.82 \\
C22:2 & 19.41 & 9.58 \\
C22:3 & 20.73 & 5.64 \\
C22:4 & 9.18 & 6.18 \\
C22:5 & 11.94 & 6.09 \\
C22:6 & 5.77 & 5.10
\end{tabular}

Energy-minimized three-dimensional compounds were prepared (Fig. 3) using Insight II (Accelrys, San Diego, CA, USA).

These results suggested that C22-fatty acids could bind to and interact with the hydrophobic region of the protein of pols and topos. We also tested whether an excess amount of a substrate DNA analog, poly $(\mathrm{rC})(100 \mu \mathrm{g} / \mathrm{ml})$, or a protein, BSA $(200 \mu \mathrm{g} / \mathrm{ml})$, could prevent the inhibitory effects of C22fatty acids. If C22-fatty acids bind to enzymes by non-specific adhesion, the addition of nucleic acid and/or protein would be expected to reduce inhibitory activity. The fact that neither poly(rC) nor BSA influenced the inhibitory effects of C22fatty acids suggests that the compound occurs selectively or binds to a specific site on the enzymes and not to the nucleic acid.

Three-dimensional structure modeling of linear-chain C22fatty acids. Cis-13,16-docosadienoic acid (C22:2) was the strongest pol and topo inhibitor among the linear-chain C22fatty acids tested. Therefore, we carried out three-dimensional structure modeling analysis of the linear-chain C22-fatty acids, and speculated the enzyme model with the fatty acid. The energy-minimized three-dimensional linear-chain fatty acids by computer modeling are shown in Fig. 3. The hydrocarbon chain in the saturated and trans-unsaturated C22-fatty acid molecule was linear because of no or little width. Di- and more unsaturated C22-fatty acids of the cis-configuration lost molecular length and formed width because the hydrocarbon chains in their fatty acids fold up at the double bonds position in the cis-configuration. The molecular lengths and widths of linear-chain C22-fatty acids are compared in Table I. The hydrocarbon chain in the saturated and trans-unsaturated fatty acid molecules was linear, the longest length being $28.86 \AA$ (Table I), and very weakly inhibited the activity of pols and topos (Figs. 1 and 2). Mono- and more unsaturated C22-fatty acids of cis-configuration reduced molecular length, and more than four unsaturated fatty acids formed a ballshaped curve. Cis-13,16-docosadienoic acid (C22:2) and cis13,16,19-docosatrienoic acid (C22:3) strongly inhibited the activity of topos and pols, and a length of approximately 19-21 $\AA$ fatty acid was required for inhibition. Furthermore, the molecular widths in C22-fatty acids are compared in Fig. 3B. Cis-13,16-docosadienoic acid (C22:2), which was the strongest inhibitor among C22-fatty acids, formed a Vshaped curve, and the molecular width was the largest (9.58 $)$
(Fig. 3B and Table I). The molecular width (>7 $\AA$ ) in the fatty acids also appeared to be important for enzyme inhibition.

From the results above, the inhibitory effects of C22-fatty acids on the activity of pols and topos could occur by binding between the enzyme protein and the fatty acid. The substrate DNA binding sites of the enzymes had a pocket which can bind to the fatty acid. If their fatty acid-binding site structures were three-dimensionally similar, the substrate DNA binding site of the topos would also have a pocket which can bind to cis-13,16-docosadienoic acid (C22:2), like pols. The size of the pocket must have sufficient length and width to join to the cis-13,16-docosadienoic acid (C22:2) molecule (i.e., length of $19.41 \AA$ and width of $9.58 \AA$ ). The pocket must consist of hydrophilic amino acids to bind to the substrate DNA and a hydrophobic polypeptide sheet to bind to the hydrocarbon chain of fatty acid. Moreover, the carboxyl group of cis-13,16-docosadienoic acid (C22:2) must bind to the hydrophilic amino acid in the pocket in competition with substrate DNA, because the free carboxyl group was important for enzyme inhibition. These are predictable for future X-ray crystal and NMR analyses of the complex of enzymes and fatty acids.

\section{Discussion}

PUFAs have been documented to inhibit or even prevent cancer. Epidemiological evidence strongly links fish oil [rich in DHA (C22:6)] with low incidences of several types of cancer (17-22). The inhibitory effects of PUFAs including C22-fatty acids such as DPA (C22:5) and DHA on cancer development and progression are supported by studies using cultured cells and animal models (23-30). In this study, PUFAs such as unsaturated C22-fatty acids were investigated for the inhibitory activity of DNA metabolic enzymes such as pols and topos, and cis-13,16-docosadienoic acid (C22:2) was a stronger inhibitor than DPA and DHA. Therefore, this fatty acid could be an anti-cancer fatty acid among PUFAs.

Several anti-cancer agents in clinical use have been shown to be potent inhibitors of topos. For example, adriamycin (doxorubicin), amsacrine (m-AMSA) and ellipticine have been demonstrated to show significant activity as inhibitors of topo II (31). The plant alkaloid camptothecin and its synthetic derivatives such as CPT-11 are extensively studied topo I inhibitors (32). All of these agents inhibit the rejoining reaction of topos by stabilizing a covalent topo-DNA complex termed the 'cleavable complex'. Thus, no C22-fatty acids such as cis-13,16-docosadienoic acid (C22:2) bound to the dsDNA, suggesting that it must inhibit enzyme activity by interacting with the enzymes directly, and that C22-fatty acids were of the non-cleavable complex type of topo II inhibitors.

As described in the Introduction, both pols and topos are attractive targets for cancer chemotherapy $(3,6-8)$; therefore, clarifying the molecular mechanism of their inhibition should provide clues to create an ideal artificial cancer chemotherapy agent. Our studies may provide insight into the mechanism of this effect, because cis-configurated di-unsaturated linear-chain C22-fatty acids, cis-13,16-docosadienoic acid (C22:2), strongly inhibited the activity of human topos, and because the inhibition mode of topos by C22-fatty acids was almost the same as that of pols. Therefore, the three-dimensional structural information 
of linear-chain C22-fatty acids concerning inhibition described in this report may facilitate the computer design of new molecular probes capable of functioning as anti-neoplastic agents.

\section{Acknowledgments}

We are grateful for the donations of calf pol $\alpha$, rat pol $\beta$, human pols $\delta$ and $\varepsilon$, and human pol $\lambda$ by Dr M. Takemura of Mie University (Tsu, Japan), Dr A. Matsukage of Japan Women's University (Tokyo, Japan), Drs K. Sakaguchi, N. Shimazaki and O. Koiwai of Tokyo University of Science (Chiba, Japan), respectively. This work was supported in part by a Sasakawa Scientific Research Grant (to Y.N.) from the Japan Science Society, and by a Grant-in-aid for Kobe-Gakuin University Joint Research (A) (to H.Y. and Y.M.). Y.M. acknowledges Grants-in-aid from the Uehara Memorial Foundation (Japan), the Mochida Memorial Foundation for Medical and Pharmaceutical Research (Japan), and the Nakashima Foundation (Japan).

\section{References}

1. Mizushina Y, Yagi H, Tanaka N, Kurosawa T, Seto H, Katumi K, Onoue M, Ishida H, Iseki A, Nara T, Morohashi K, Horie T, Onomura Y, Narusawa N, Aoyagi N, Takami M, Yamaoka M, Inoue Y, Matsukage A, Yoshida S and Sakaguchi K: Screening of inhibitor of eukaryotic DNA polymerases produced by microorganisms. J Antibiot 49: 491-492, 1996.

2. Mizushina Y, Tanaka N, Yagi H, Kurosawa T, Onoue M, Seto H, Horie T, Aoyagi N, Yamaoka M, Matsukage A, Yoshida S and Sakaguchi K: Fatty acids selectively inhibit eukaryotic DNA polymerase activities in vitro. Biochim Biophys Acta 1308: 256-262, 1996.

3. Kornberg A and Baker TA: DNA Replication. 2nd edition. W.H. Freeman, New York, pp197-225, 1992.

4. Hubscher U, Maga G and Spadari S: Eukaryotic DNA polymerases. Annu Rev Biochem 71: 133-163, 2002.

5. Wang JC: DNA topoisomerases. Annu Rev Biochem 54: 665-697, 1998.

6. Liu LF: DNA topoisomerase poisons as antitumor drugs. Annu Rev Biochem 58: 351-375, 1989.

7. Chakraborty AK and Majumder HK: Mode of action of pentavalent antimonials: specific inhibition of type I DNA topoisomerase of Leishmania donovani. Biochem Biophys Res Commun 152: 605-611, 1988.

8. Ray S, Hazra B, Mittra B, Das A and Majumder HK: Diospyrin, a bisnaphthoquinone: a novel inhibitor of type I DNA topoisomerase of Leishmania donovani. Mol Pharmacol 54: 994-999, 1998.

9. Boege F, Straub T, Kehr A, Boesenberg C, Christiansen K, Andersen A, Jakob F and Kohrle J: Selected novel flavones inhibit the DNA binding or the DNA religation step of eukaryotic topoisomerase I. J Biol Chem 271: 2262-2270, 1996.

10. Fortune JM and Osheroff N: Merbarone inhibits the catalytic activity of human topoisomerase IIalpha by blocking DNA cleavage. J Biol Chem 273: 17643-17650, 1998.

11. Bridewell DJ, Finlay GJ and Baguley BC: Differential actions of aclarubicin and doxorubicin: the role of topoisomerase I. Oncol Res 9: 535-542, 1997.

12. Tamai K, Kojima K, Hanaichi T, Masaki S, Suzuki M, Umekawa $\mathrm{H}$ and Yoshida S: Structural study of immunoaffinitypurified DNA polymerase $\alpha$-DNA primase complex from calf thymus. Biochim Biophys Acta 950: 263-273, 1988.
13. Date T, Yamaguchi M, Hirose F, Nishimoto Y, Tanihara K and Matsukage A: Expression of active rat DNA polymerase B in Escherichia coli. Biochemistry 27: 2983-2990, 1988.

14. Oshige M, Takeuchi R, Ruike R, Kuroda K and Sakaguchi K: Subunit protein-affinity isolation of Drosophila DNA polymerase catalytic subunit. Protein Expr Purif 35: 248-256, 2004.

15. Shimazaki N, Yoshida K, Kobayashi T, Toji S, Tamai T and Koiwai O: Over-expression of human DNA polymerase lambda in E. coli and characterization of the recombinant enzyme. Genes Cells 7: 639-651, 2002.

16. Ogawa A, Murate T, Suzuki M, Nimura Y and Yoshida S: Lithocholic acid, a putative tumor promoter, inhibits mammalian DNA polymerase B. Jpn J Cancer Res 89: 1154-1159, 1998.

17. Bang $\mathrm{OH}$ and Dyerberg J: Plasma lipids and lipoproteins in Green-landic west coast Eskimos. Acta Med Scand 192: 85-94, 1972 .

18. Vogel VG and McPherson RS: Dietary epidemiology of colon cancer. Hematol Oncol Clin North Am 3: 35-63, 1989.

19. Kaizer L, Boyd NF, Kriukov V and Tritchler D: Fish consumption and breast cancer risk: an ecological study. Nutr Cancer 12: 61-68, 1989.

20. Schloss I, Kidd MS, Tichelaar HY, Young GO and O'Keefe SJ: Dietary factors associated with a low risk of colon cancer in coloured west coast fishermen. S Afr Med J 87: 152-158, 1997.

21. Berg JP, Glattre E, Haldorsen T, Hostmark AT, Bay IG, Johansen AF and Jellum E: Longchain serum fatty acids and risk of thyroid cancer: a population-based case-control study in Norway. Cancer Causes Control 5: 433-439, 1994.

22. Terry P, Lichtenstein P, Feychting M, Ahlbom A and Wolk A: Fatty fish consumption and risk of prostate cancer. Lancet 357: 1764-1766, 2001.

23. Noguchi M, Minami M, Yagasaki R, Kinoshita K, Earashi M, Kitagawa H, Taniya T and Miyazaki I: Chemoprevention of DMBA-induced mammary carcinogenesis in rats by low-dose EPA and DHA. Br J Cancer 75: 348-353, 1997.

24. Rose DP, Connolly JM and Coleman M: Effect of omega-3 fatty acids on the progression of metastases after the surgical excision of human breast cancer cell solid tumors growing in nude mice. Clin Cancer Res 2: 1751-1756, 1996.

25. Jenski LJ, Zerouga $M$ and Stillwell W: Omega-3 fatty acidcontaining liposomes in cancer therapy. Proc Soc Exp Biol Med 210: 227-233, 1995.

26. Norrish AE, Skeaff CM, Arribas GL, Sharpe SJ and Jackson RT: Prostate cancer risk and consumption of fish oils: a dietary biomarker-based case-control study. Br J Cancer 81: 1238-1242, 1999.

27. Calviello G, Palozza P, Maggiano N, Franceschelli P, Di Nicuolo F, Marcocci ME and Bartoli GM: Effects of eicosapentaenoic and docosahexaenoic acids dietary supplementation on cell proliferation and apoptosis in rat colonic mucosa. Lipids 34: S111, 1999.

28. Calviello G, Palozza P, Maggiano N, Piccioni E, Franceschelli P, Frattucci A, Di Nicuolo F and Bartoli GM: Cell proliferation, differentiation, and apoptosis are modified by $n-3$ polyunsaturated fatty acids in normal colonic mucosa. Lipids 34: 599-604, 1999.

29. Calviello G, Palozza P, Piccioni E, Maggiano N, Frattucci A, Franceschelli P and Bartoli GM: Dietary supplementation with eicosapentaenoic and docosahexaenoic acid inhibits growth of Morris hepatocarcinoma 3924A in rats: effects on proliferation and apoptosis. Int J Cancer 75: 699-705, 1998.

30. Madhavi N and Das UN: Effect of n-6 and n-3 fatty acids on the survival of vincristine sensitive and resistant human cervical carcinoma cells in vitro. Cancer Lett 84: 31-41, 1994.

31. DeMarini DM and Lawrence BK: Prophage induction by DNA topoisomerase II poisons and reactive-oxygen species: role of DNA breaks. Mutat Res 267: 1-17, 1992.

32. Taniguchi K, Kohno K, Kawanami K, Wada M, Kanematsu T and Kuwano M: Drug-induced down-regulation of topoisomerase I in human epidermoid cancer cells resistant to saintopin and camptothecins. Cancer Res 56: 2348-2354, 1996. 